\title{
Palm Vein Extraction and Matching For Personal Identification
}

\author{
Mr. Vishal U. Bhosale ${ }^{1}$, Mr. Onkar S. Kale ${ }^{2}$, Mr. Mahesh W. Pawar ${ }^{3}$, \\ Mr. Roshan R. Patil ${ }^{4}$, Mr. Pritam S. Patil ${ }^{5}$, Prof Mrs. Sonali Madankar ${ }^{6}$. \\ 1,2,3,4,5,6 (IT Dept., PVPIT, Pune, India)
}

\begin{abstract}
The most common method used for authentication is Textual passwords. But Textual passwords are in risk to eves dropping, dictionary attacks, social engineering and shoulder surfing. Biometric passwords are introduced as alternative techniques to textual passwords. Biometrics is used for human recognition which consists of authentication, verification and recognition. A CCD camera will capture the image and this image will process through various algorithms. In future this image will be compared for personal authentication. In this paper, three algorithms are proposed to extract the images taken by CCD camera.
\end{abstract}

Keywords: Biometric, palm vein pattern, ROI extraction, feature extraction, matching, Vascular Pattern, NearInfrared Image, Grayscaling, Thresholding, Thinning.

\section{Introduction}

The most common method used for authentication is textual password. The vulnerabilities of this method like eves dropping, dictionary attack, social engineering and shoulder surfing are well known. Arbitrary and lengthy passwords can make the system secure. But the main problem is the difficulty of remembering those passwords. Studies have shown that users tend to pick short passwords or passwords that are easy to remember. Unfortunately, these passwords can be easily guessed or broken. The alternative techniques are biometrics and graphical passwords. But these two techniques have their own disadvantages. There are many graphical passwords schemes that are proposed in the last decade. But most of them suffer from shoulder surfing which is becoming quite a big problem. There are graphical passwords schemes that have been proposed which are resistant to shoulder-surfing but they have their own drawbacks like usability issues or taking more time for user to login. Palm vein recognition system consists of four key steps: Infrared palm images capture Detection of Region of Interest (ROI) and pre-processing and Palm vein pattern extraction, feature extraction and feature matching.

\section{Related Works}

Debnath Bhattacharyya and his colleagues proposed that an Image Analysis technique for Vascular Pattern of Hand Palm, which in turn leads towards Palm Vein Authentication of an individual. Near-Infrared Image of Palm Vein pattern is taken and passed through three different processes or algorithms to process the Infrared Image in such a way that the future authentication can be done accurately or almost exactly. These three different processes are: a. Vascular Pattern Marker Algorithm (VPMA); b. Vascular Pattern Extractor Algorithm (VPEA); and c. Vascular Pattern Thinning Algorithm (VPTA). The resultant Images will be stored in a Database, as the vascular patterns are unique to each individual, so future authentication can be done by comparing the pattern of veins in the palm of a person being authenticated with a pattern stored in a database.[1] Yingbo Zhou and Ajay Kumar proposed two new approaches to improve the performance of palm vein based identification systems. They systematically compare previously proposed palm vein identification approaches with their proposed ones on two different databases that are acquired with the contactless and touch based imaging setup. They evaluate the performance, improvement both the verification and recognition scenarios and analyze the influence of enrollment size on performance. The rigorously experimental results presented in this paper on the database of 100 and 250 subjects, consistently conforms the superiority of the proposed approach in both the verification and recognition scenarios.[2]

Mona A. Ahmed and Hala M. Ebiied present the Analysis of palm vein pattern recognition algorithms, techniques, methodologies and systems. They discussed some technical aspects of recent approaches for the following processes detection of ROI (Region of Interest), segment o palm vein pattern, feature extraction and matching. Their result shows that, there is no benchmark database exists for palm vein recognition. For all processes, there are many machine learning techniques with very high accuracy.[3]. 
TABLE1: Various analyses by different authors

\begin{tabular}{|l|l|l|l|}
\hline Authors & Pattern Extraction & Feature Extraction & Matching \\
\hline Hassan et al[4] & & SITF & Linear Vector Quantization \\
\hline Zhou and Kumar[5] & Histogram equalization & Hessian Phase & $\begin{array}{l}\text { Neighborhood } \\
\text { Random Transform }\end{array}$ \\
\hline Ladoux et al[6] & Gaussian low-pass 51x51 & SITF & $\begin{array}{l}\text { Euclidean } \\
\text { SITF descriptance between }\end{array}$ \\
\hline $\begin{array}{l}\text { Mirmohama dsadeghi and } \\
\text { Drygajlo } \\
\text { [7] }\end{array}$ & $\begin{array}{l}\text { Local Binary Patterns and } \\
\text { Local Derivative Pattern }\end{array}$ & LBPs and high order LDPs & Histogram intersection \\
\hline
\end{tabular}

\section{Project Work}

The project illustrated in this paper is entirely based on the idea of palm vein authentication. Here, the main objective of this project is to provide security to the confidential areas such as access room or locker rooms through biometric passwords. It includes 3 phases: registration, primary level authentication, secondary level authentication (draw-a-secret). The process of figuring out the validate person is accomplished in the following manner:

\subsection{Registration}

Here we register the new user by accepting the details of new user like user id, user name and palm vein image.

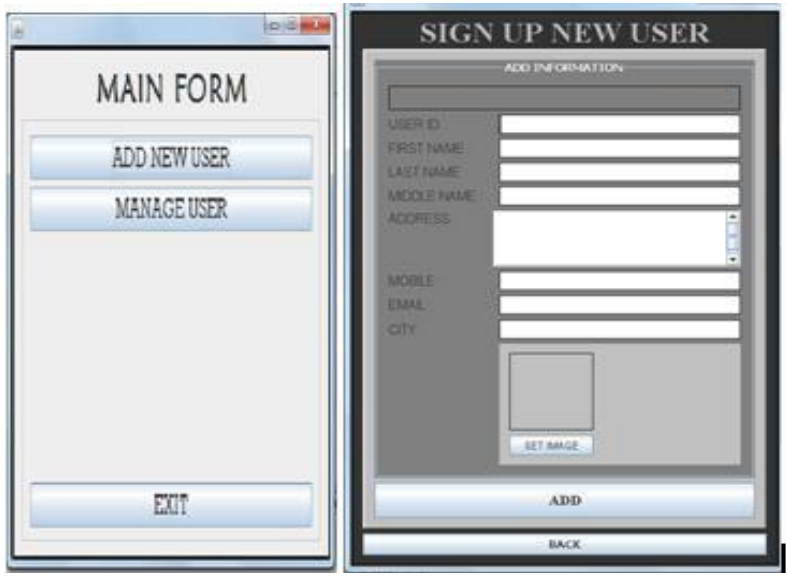

Fig 1: Registration Screen

3.2 Image Capture

Here we take image of palm vein of the person for authentication and identification purposes.

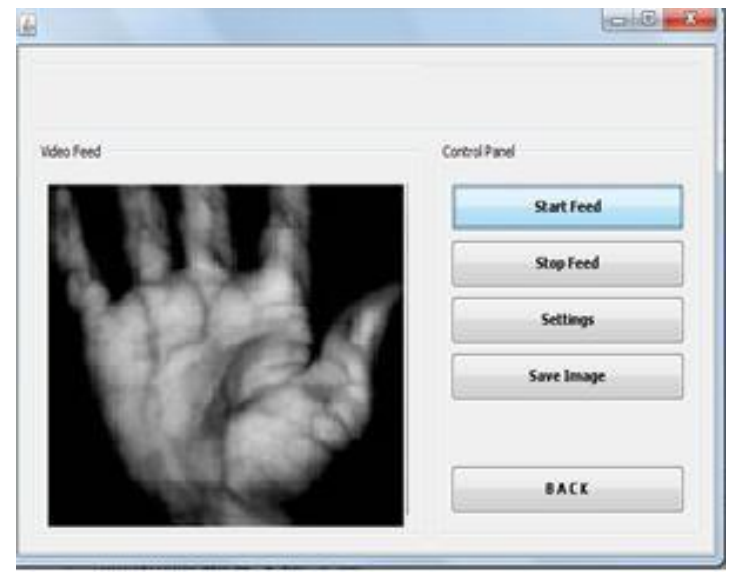

Fig 2: Image Capture

3.3 Image Processing Algorithm

In this process we capture the image taken for further processing using the three algorithms. 


\subsubsection{Pattern Marker Algorithm}

In this algorithm the image we have got from near infrared is processed, this is the actual gray scaling of our images.

\section{Algorithm}

a) Here traversal through the entire array of input image is done.

b) Read color value of each bit which is 24 bit.

c) Color value is to be split into three individual $\mathrm{R}, \mathrm{G}$ and $\mathrm{B}$ which is 8-bit values each.

d) Here conversion formula is used to calculate the grayscale component (8-bit) for given R, G and B pixels

e) Further we compose a 24-bit pixel from 8 bit gray scale value.

f) This 8 bit gray scale is stored at the same location in output image.

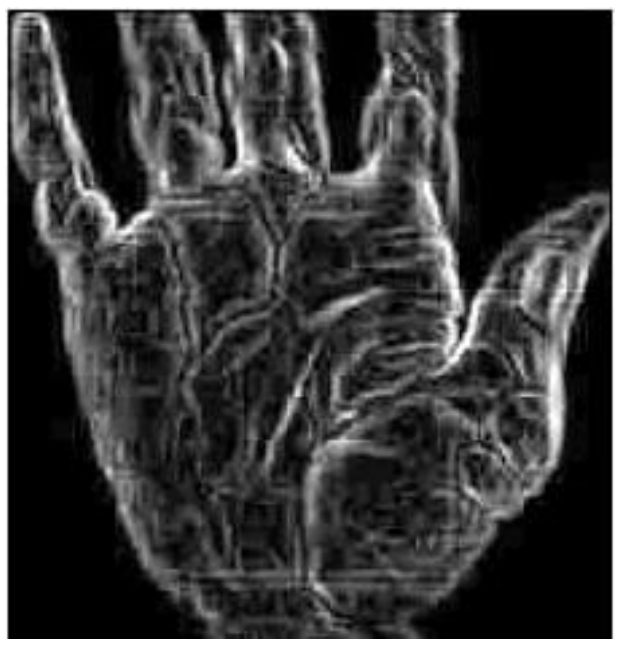

Fig.3: Grayscale Pattern

\subsubsection{Pattern Extractor Algorithm}

In Extractor Algorithm we perform Thresholding and Grayscaling. This Thresholding is the simplest image segmentation technique. Afterwards to create binary images from a grayscale image, Thresholding is used. Only black and white image can be seen in the binary image. This is used for the further processing of the image to extract only the important features and make it black or white.

\section{Algorithm:}

1) Here again we traverse through entire array of input image.

2) Covert image into gray scale reading each pixel color.

3) Now the binary output value is calculated using the pixel value which we got from thresholdingv(vthreshold value).

4) Binary pixel value is stored at the same location as that of output image.

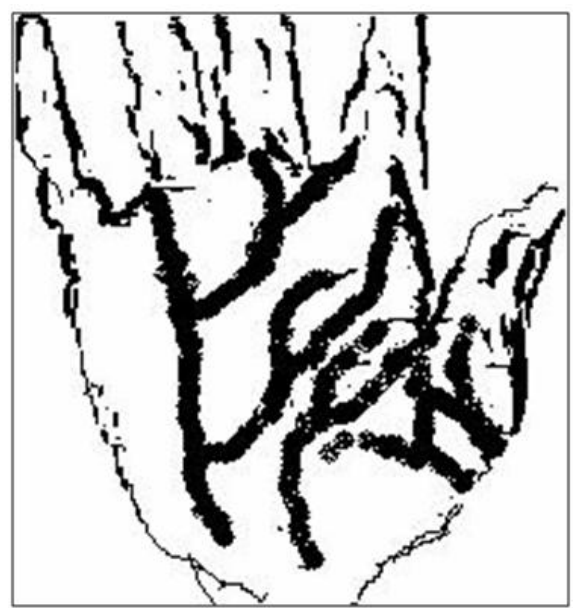

Fig.4: Threshold Pattern 


\subsubsection{Pattern Thinning Algorithm:}

The image which is extracted undergoes thinning using the Thinning algorithm. Now this thinned image is used for the authentification and identification purposes. Basically to decrease the geometric value of the object image thinning is done. Stentiford Algorithm of thinning is used for thinning the algorithm. The resultant image is shown as below

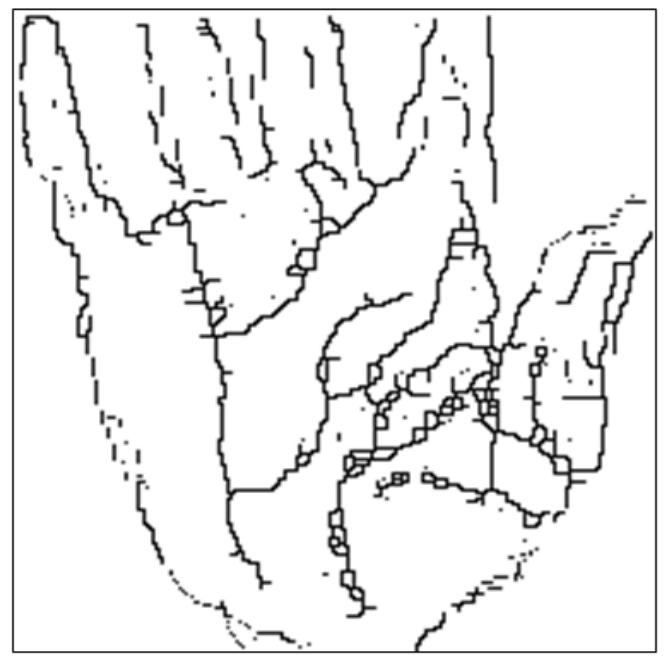

Fig.5: Thinned Pattern

3.4. Adding Thinned Image to the Database

In this step we add the thinned pattern to the database

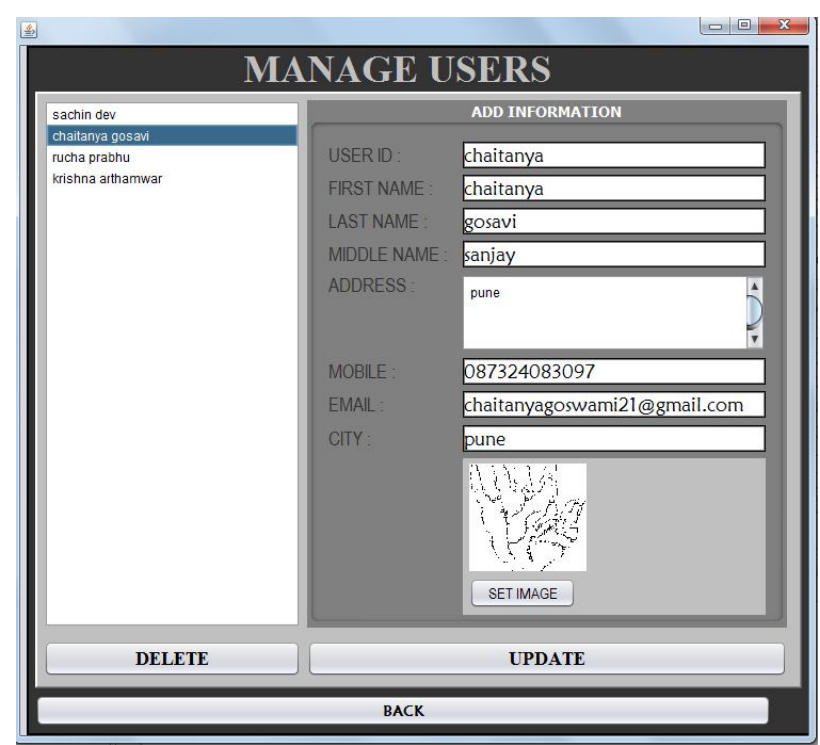

\subsection{Authentication}

We are working on the authentication process

\section{Conclusion}

We use three different algorithms for processing Palm Vein Pattern Image of an individual. This processed Image will be used for authentication of a person.

\section{Acknowledgements}

The authors would like to thank project guide Prof Mrs. S.B.MADANKAR PVPIT, Pune (India).for their guidelines and involving in research. 


\section{Journal Papers:}

\section{References}

[1]. Debnath Bhattacharya, Poulami Das, Taihoon Kim, Samir Bandopadhyay, "Vascular Pattern Analysis towards Pervasive Palm Vein Authentication", Journal of Universal Computer Science, vol. 15, no. 5 (2009),

[2]. Yingbo Zhou, Ajay Kumar, Senior member of IEEE, "Human identification using palm vein images", IEEE transactions on information forensics and security ,vol. 6, no. 4 (2011)

[3]. Mona A. Ahmed, Hala M. Ebid, El-Sayed M. Horbaty, Abdel-Badeeh M. Salem, Ain Shams University, Cairo, Egypt,"Analysis of palm vein pattern recognition algorithms and systems, International Journal of biomedical informatics and e-health, vol. 1, no 1 (2013).

[4]. Hassan Soliman, Abdelnasser Saber Mohamed and Ahmed Atwan, Feature Level Fusion of Palm Veins and Signature Biometrics , International Journal of Video \& Image Processing and Network Security IJVIPNS-IJENS Vol: 12 No: 01, pp.28-39, 2012.

[5]. Yingbo Zhou and Ajay Kumar,"Contactless Palm Vein Identification using Multiple Representations", in ${ }^{\text {th }}$ IEEE international conference on biometrics: Theory applications and systems (BTAS), pp. 1-6, September 2010.

[6]. Pierre-Olivier Ladoux, Christophe Rosenberger and Bernadette Dorizzi , "Palm Vein Verification System based on SIFT matching", Third International Conference on Advances in Biometrics, pp. 1290-1298, June 2009.

[7]. Leila Mirmohamad sadeghi and Andrzej Drygajlo, "Palm Vein Recognition with Local Binary Patterns and Local Derivative Patterns", International Joint Conference on Biometrics, pp. 1-6, 2011. 